\title{
Knowledge, Awareness, and Attitude of Doctors and Patients regarding Informed Consent to Medical Procedures in Papua New Guinea
}

\author{
Alfred P. Minei ${ }^{1}$, Rachelyn Ann S. Araña ${ }^{2}$ and Ronald R. Roldan Jr. ${ }^{2}$ \\ 1. School of Law, University of Papua New Guinea, Post Office Box 126, Waigani, National Capital District, Papua New Guinea \\ 2. Institute of Statistics, College of Arts and Sciences, University of the Philippines Los Baños, Los Baños, Laguna 4031, Philippines
}

\begin{abstract}
This study explores how doctors and patients in Papua New Guinea (PNG) perceive informed consent in medical settings. Doctors and patients from National Capital District and Central Province who responded to the survey were the participants of the study. Researchers asked the participants to fill out questionnaires regarding their knowledge about informed consent for each group, namely, the doctors and participants. From those who responded, six randomly selected participants were chosen to join the focus group discussion which aimed to get experiences from the doctors and patients regarding medical procedures. Results show that both doctors and patients lack knowledge of the legalities of informed consent. Based on the experiences of doctors, they do not use consent forms when seeking permission from patients. Patients, on the other hand, do not see consent forms as important and only served as a formality. Customs, culture surrounding PNG were found to have an impact on how patients perceived informed consent.
\end{abstract}

Key words: Informed consent, surveys, focus group discussion, PNG.

\section{Introduction}

Medical (or health) care professionals are held to a specific standard of informed consent when treating their patients. Patients, on the other hand, have the right to decide on the medical treatment they will receive based on the doctor's diagnosis. In the process of communication between the patient and doctor in terms of the treatments, the treatment that the patient must undergo should result in an explicit agreement. This is under the assumption that patients understand and agree to the potential consequences of the medical care they will receive.

There are challenges that usually occur when obtaining informed consent in medical settings. In India [1], the physician is held in very high esteem.

This scenario is also common in Japan [2], where

Corresponding author: Alfred Minei, PhD candidate, research fields: law and medicine, and patients typically proceed with treatment regimens as recommended by the practitioners without asking about the possible effects of the procedure. patients rarely ask questions about their treatment or treatment options. The effect of this high level of trust between a patient and physician may pose ethical issues that both parties might not readily know. Ethical issue of consent has generated the most debate globally regarding healthcare needs [3]. The consent procedures seem inadequate as a means for the expression of autonomous choice and the ethical standing can be called into question. Lack of informed consent can reinforce a claim of medical malpractice where the case is otherwise weak. Demonstration of a real conducted informed consent process not only protects the physician from exposure to liability but also increases the patient's autonomy in decision concerning health and encourages compliance with treatment.

The concept of informed consent has not received a great deal of attention in developing countries. In Papua New Guinea (PNG), informed consent is given in a form filled or signed by patients or guardians in operating theatres at the hospitals. This being the case, 
then informed consent is a duty owed to the patient and does not properly support the notion that informed consent is a patient's right. It fails to grasp the true meaning of informed consent. Informed consent is often obtained without the patient's understanding and this may increase the chances of exploitation against inappropriate discriminatory negligent by the healthcare professionals for patients. It is believed that, where beliefs and opinions of individuals are taken into account on informed consent to medical treatment, patients make their decisions which affect their healthcare needs, and or a breach of patient's right by a physician is a malpractice by the healthcare professional $[4,5]$.

This study investigates the process of informed consent and determines the doctors' and patients' awareness, practices, attitude, understanding and perceptions of the legal nature of informed consent in PNG [6]. In addition, the study describes the reasons about the perceptions on how the study subsets view and practice informed consent.

\section{Methodology}

\subsection{Research Design}

The design employed in this study is a mixture of qualitative and quantitative research designs. It is also an exploratory study on how doctors and patients perceive informed consent to medical procedures. Surveys among doctors and patients were done to determine the knowledge, awareness, and attitude towards informed consent to medical procedures. Focus group discussions (FGD) for each group of respondents were conducted to validate and triangulate the quantitative results found in the surveys.

\subsection{Participants}

The study was conducted in the hospitals and clinics in the National Capital District and Central Province of PNG. There are 19 doctors and 29 patients who participated in the study.

About $53 \%$ of the doctors are male and $80 \%$ are married. The youngest and oldest doctors are 27 and 56 years old, respectively. They are usually 42 years old, on the average. In terms of working experience, they have been practicing for an average of 17 years, with 6 and 34 years as the shortest and longest working experience. The number of female patients they encounter in a week varies from 20 to 21. They have served female patients in numbers as low as five and as high as 80 in a week.

Forty eight percent of the patients are female. Majority (58.6\%) of them are married. About $75 \%$ of them are at least high school graduate. Their age deviates from 38 years old by 14 years. The youngest and oldest are 25 and 68 years old respectively.

\subsection{Instruments and Materials}

Two sets of survey questionnaires and interview guides were created to determine the knowledge, awareness, and attitude of doctors and patients towards informed consent. Some items in the survey questionnaires were adapted from the studies of Chima [7], Rajesh et al. [8], Ashraf et al. [9], and Parmar et al. [10].

The questionnaire given to doctors focused on how they deal with patients seeking medical treatment, their knowledge of how informed consent is given as well as the legal aspects of informed consent. On the other hand, the questionnaire given to patients is focused on their knowledge and legal aspects of informed consent, information they want to know before signing the consent form, perception on who is to decide in terms of medical treatment and some factors affecting their capacity in signing consent forms were included.

The interview exploratory guides were used in the focus group discussion. The questions focused on reasons of doctors pertaining to how they view and practice informed consent. On the other hand, the questions for the patients focused on their experiences when seeking medical treatment and hindrances they encounter when making decisions about the medical 
procedure they need to undergo.

\subsection{Data Collection}

Before the conduct of the survey, pre-testing of questionnaires was done with five doctors, medical and law students, and patients at the university clinic. All items were checked for consistency of answer of the participants and added additional responses to one question. No major revisions were made in the questionnaires.

In the actual survey, consent was asked for and granted by hospitals/clinics in the National Capital District and Central Province. The participants who were willing to answer the self-administered questionnaire were considered participants of this study. About 35 survey questionnaires for each group were distributed in these hospitals and clinics.

After the conduct of the survey, a focus group discussion was conducted for each group of participants. Random samples of respondents were selected from those who participated in the survey in each group. Those randomly selected respondents were invited to join the focus group discussion. The session for doctors and patients lasted for 90 and 130 minutes, respectively. The focus group discussion was audio-taped and digitally recorded to aid in transcription.

\subsection{Data Analysis}

To determine the knowledge, and awareness of doctors and patients about informed consent and its legal aspects, frequencies and percentage were generated.

To determine the knowledge of patients and doctors on the legal aspects of informed consent, frequencies and percentages were computed for each statement.

To describe the practices of doctors in obtaining informed consent and patients' experience in getting medical treatment, responses of the doctors and patients from the focus group discussions were transcribed and summarized. From these responses, themes were constructed.

To compare the perception of doctors and patients towards informed consent, proportions of doctors and patients who agreed on each statement were compared. The responses from the focus group discussion were integrated in the comparison.

\section{Results and Discussion}

\subsection{Knowledge and Practice of Doctors on Getting Consents from Patients}

Majority of doctors (78.95\%) said that informed consent should be applied to all procedures to be done with the patient while $10.53 \%$ of them answered that informed consent should only be applied to critical cases (Table 1).

In terms of the content of the consent form, more than $80 \%$ agreed that patient's education, recording of the consent, and patient's and doctor's signatures should be on the form. Only about 39\% of the doctors cared to include their evaluation of competence in the form. In terms of age of patients who can give valid informed consent, it can be observed that there are varying responses given by the doctors. Most of them (42.11\%) said patients aged above 17 are the ones who can give valid consent. One doctor proposed it to be above 18 and include the patient's education in the criteria. In terms of who is to give the informed consent, all doctors agreed that it should come from the patient. However, there were notes coming from the doctors that for minors, parents should be involved in this decision. Also, they added that if the patient cannot provide consent because of unconsciousness or not in the right state of mind, husband, father, or relatives can decide for them.

In terms of what time to get the consent, more than $90 \%$ of them answered: in the ward or clinic. Some doctors answered in the operating room especially if these are emergency cases. Some suggested it be 
given at the admission counter for straightforward

cases.

Table 1 Knowledge on informed consent of doctor respondents.

\begin{tabular}{|c|c|}
\hline Knowledge & Percentage of doctors \\
\hline \multicolumn{2}{|l|}{ When is informed consent applied? } \\
\hline All procedures & 78.95 \\
\hline Elective ones & 10.53 \\
\hline Critical cases only & 10.53 \\
\hline \multicolumn{2}{|l|}{ Content of consent form } \\
\hline Evaluation of competence & 38.89 \\
\hline Patient education & 84.21 \\
\hline Recording of consent & 84.21 \\
\hline Patient’s signature & 89.47 \\
\hline Doctors' signature & 89.47 \\
\hline Witness & 11.12 \\
\hline Stamp/date & 11.12 \\
\hline \multicolumn{2}{|l|}{ Age of those who can give valid informed consent } \\
\hline All ages & 36.84 \\
\hline Patients aged above 17 & 42.11 \\
\hline Depending on clinical judgement of maturity & 10.53 \\
\hline Above 18 and depends on level of education & 5.26 \\
\hline Special consideration on people with disability & 5.26 \\
\hline \multicolumn{2}{|l|}{ Which time to obtain informed consent? } \\
\hline At the admission counter & 38.89 \\
\hline In the ward/clinic & 94.44 \\
\hline In the operation room & 44.44 \\
\hline The night before the procedure & 77.78 \\
\hline
\end{tabular}

\subsection{Knowledge and Practices of Patients towards} Informed Consent

Patients were asked about their knowledge on giving consent to doctors on medical procedure and the possible factors that can hinder them in giving such consent. Their responses are presented in Table 2. About $86 \%$ of the patients are aware of what an informed consent is and about $93 \%$ of them believe that informed consent is important before doing a medical procedure. Despite this great proportion of all answers coming from the patient respondents in terms of knowledge about informed consent, it cannot be denied that there are still patients who are not aware of what an informed consent is and its importance in medical treatment.

Among the factors that might hinder patients in signing a consent form, family and being unable to understand the procedure are the top reasons. About $40 \%$ of the patients also considered their spouses, religious views, and language barrier as factors that they consider when signing the forms. These are also the experience taken from the focus group discussion interviews, as patients expressed... "I like to be seen by a doctor. I prefer a female doctor because I am a female and... If a male doctor; I want my husband or family members to accompany me to talk to the doctor... My culture/custom required me to do and say certain things, respect for my husband is paramount important. Follow the custom that... no man is to see a woman...”

"The treatment cost is important. My husband and family must know because they will assist me..."

Practices of patients when they seek medical 
treatment are presented in Table 3. It can be observed healers where 34\% of them usually go when sick. In that more than about $66 \%$ of the patients chose to go to doctors when they are sick, followed by traditional

Table 2 Knowledge of patients about informed consent to medical procedures.

\begin{tabular}{ll}
\hline Do you know what an informed consent to medical procedure is? & Percentage of patients \\
\hline Yes & 86.21 \\
No & 13.79 \\
\hline Do you think informed consent is important before doing a medical procedure? & \\
\hline Yes & 93.1 \\
No & 6.9 \\
\hline Who/What can hinder you from signing a consent form? & \\
\hline Spouse & 41.38 \\
Family & 58.62 \\
Religious views & 44.83 \\
Traditional beliefs/customs & 37.93 \\
Language barrier & 41.38 \\
Do not understand & 51.72 \\
\hline
\end{tabular}

Table 3 Practices of patients when seeking medical care.

\begin{tabular}{ll}
\hline When sick, who do you usually seek for medication/check-up? & Percentage of patients \\
\hline Doctors & 65.52 \\
Traditional Healers & 34.48 \\
\hline Who can sign consent form/decide for medical procedure when too sick? & \\
\hline Family & 42.86 \\
Healer/Doctor & 21.43 \\
Next of kin & 7.14 \\
No one & 7.14 \\
Spouse & 14.29 \\
\hline Strategies adopted if the procedure was not understood & \\
\hline I ask again & 92.86 \\
Nothing & 3.57 \\
Others & 3.57 \\
\hline
\end{tabular}

terms of understanding what the doctors have said in terms of diagnosis, $93 \%$ are able to ask the doctor to repeat what the procedure is all about. But still there are a few who chose not to say anything and leave the hospital/clinic without knowing what the procedure is all about.

Patients were also asked who can represent them when they are too sick to decide for themselves. They usually choose parents or immediate family members (42\%) to decide for the medical procedure and even sign the consent form. Interestingly, there are patients who leave their fate in the hands of the doctor even in signing for them in the consent form. There are few patients (7\%) who opt not to put their fate in the hands of anyone else aside from themselves.

In terms of the things they want to know before signing a consent form, more than $90 \%$ of them agree to check the duration of stay in the hospital after the procedure, and number of hours that will be spent in conducting the procedure. Majority of them want to know all things related to the procedure before conducting it (Table 4). There are a few (10.34\%) who do not want to know the cost before signing the consent form.

In terms of the decision-making process about the medical procedure patients would undergo, $82.76 \%$ of 
the patient respondents wanted to know every wherein majority of the patient respondents (58.62\%) information of the procedure and will decide for themselves (Table 5). However, there are instances

Table 4 Perceptions of patient's willingness to know before signing consent form.

\begin{tabular}{llll}
\hline & \multicolumn{3}{l}{ Percentage of patients } \\
\cline { 2 - 4 } & Disagree & Not sure & Agree \\
\hline Cost of procedure & 10.34 & 13.79 & 75.86 \\
Reason of procedure & 0.00 & 10.34 & 89.66 \\
Time taken by the procedure & 0.00 & 6.90 & 93.10 \\
Complications of procedure & 6.90 & 6.90 & 86.21 \\
Precautions needed to be taken before and after procedure & 6.90 & 3.45 & 89.66 \\
Limitations of quality of life after procedure & 3.45 & 13.79 & 86.76 \\
Success rate of procedure & 3.45 & 10.34 & 96.55 \\
Duration of stay at hospital after procedure & 3.45 & 0.00 & 89.66 \\
Alternative way of suggested procedure & 6.90 & 3.45 & 89.66 \\
Steps of procedure & 3.45 & 6.90 & \\
\hline
\end{tabular}

Table 5 Perceptions of patient's decision making for procedure.

\begin{tabular}{llll}
\hline & & \multicolumn{2}{c}{ Percentage of Patients } \\
\cline { 2 - 4 } & Disagree & Not sure & Agree \\
\hline I trust and abide of the decision taken by doctor on behalf of me without any & 48.28 & 24.14 & 27.59 \\
information for procedure & 34.48 & 6.90 & 58.62 \\
I want to know every information of procedure but doctor will decide finally & 30.34 & 82.76 \\
\hline I want to know every information of procedure and will decide myself & 6.90 & 10.34 \\
\hline
\end{tabular}

wanted to know every information but let the doctors decide for them. Surprisingly, there are patients (27.59\%) who do not want any information about the procedure and trust the doctor with their life.

\subsection{Knowledge of Doctors and Patients about the Legal Aspects of Informed Consent}

Several questions were asked to test the knowledge of doctors and patients on the legal side of informed consent (Fig. 1). Majority of the doctors and patients agree to all statements concerning information about informed consent. Even for statements that say signing a consent form eliminates a patient's right to compensation, informed consent is a protective shield for doctors/healers, and consent form is just a formality. Based on these responses, it can be observed that both parties have little knowledge of the importance of having a consent form. Although, there is a higher percentage of patients who think this way interestingly, both parties thought that informed consent is a protective shield for the doctors.
The results from the survey responses resonated with outcomes of the focus group discussion, as Doctor "A" said: "I am not aware of a law or laws which provide for the protection of both patients and healthcare professionals. I am aware of the medical doctor's code of practice which I rely on... when I am performing my work". Consent forms were not really being used in the workplace of the doctors as several doctor respondents confirmed.

"I am aware of consent form. I see and had used at Port Moresby General Hospital. However I have not seen or used the form lately" as said by Doctor "B".

"I would like to see a uniform consent form to be used throughout so the doctors should develop a habit of ...." responded Doctor "A".

"Most consents are taken verbally and written on the patient records, including mine as well, on the patient's records." added Doctor "B".

On the side of the patients, they generally think that once they chose to enter the hospital/clinic that is already a sign of consent. They usually use waiver 
forms for critical cases however not for all medical the illness to patients was also captured in the focus procedure.

The practice of doctors on giving information on

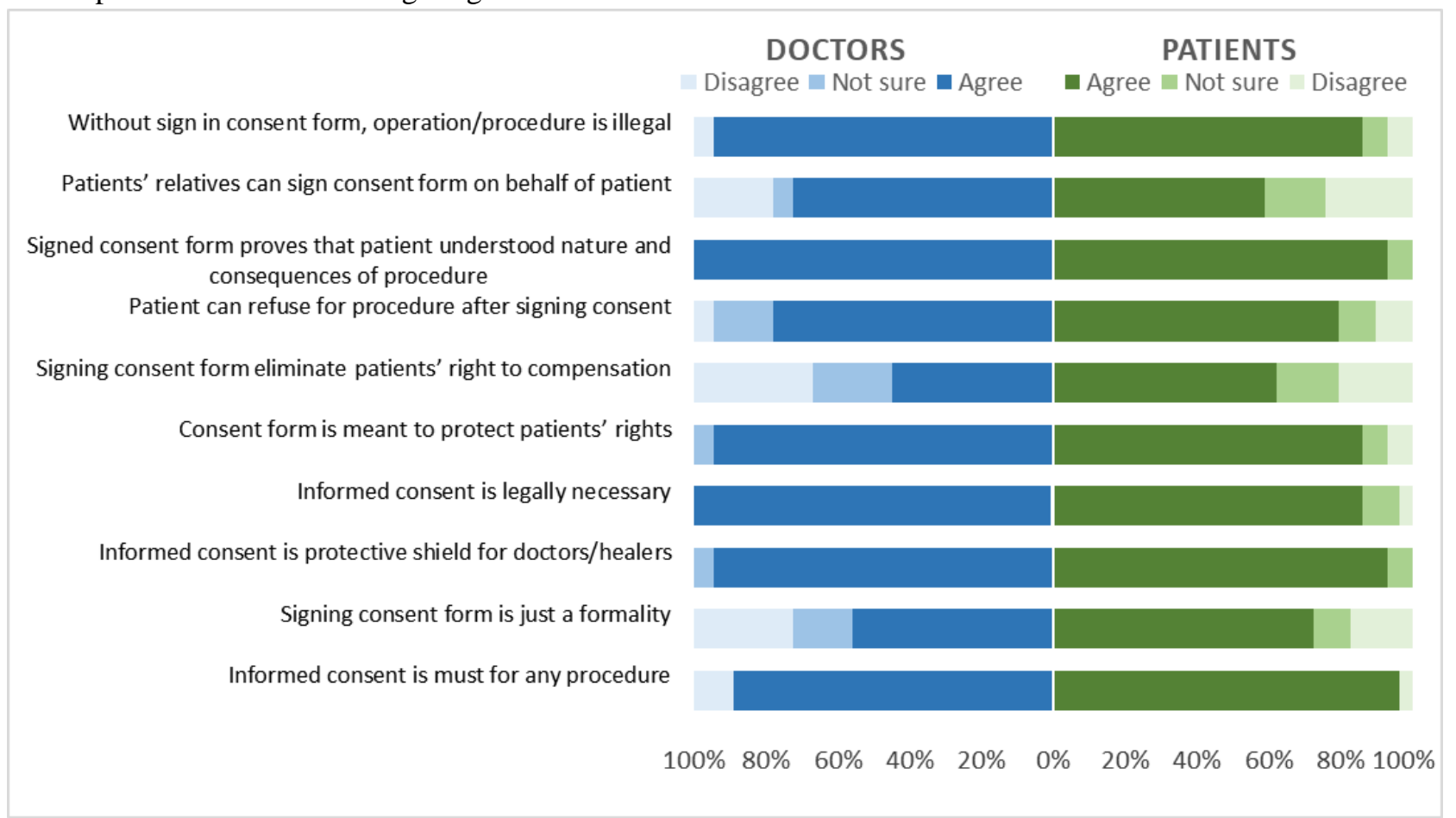

Fig. 1 Comparison of the distribution of doctors and patients on their responses about legal aspects of informed consent.

group discussion. It seemed patients wanted their records to be given to them, as one patient said:

"I have the right to know what is wrong with me. I want my records to be given to me as well. I have my rights..." On the side of the doctors, they feel there is a gap in the relationship between them and their patients, as one doctor shared, "Some patients do not feel comfortable to see a doctor. I asked a few patients why? I am told that they feel that the doctor knows it best for their treatment or procedure. The patient has to follow. I told the patient not to think that way...”.

\section{Conclusion}

Informed consent process flows between physician and patient, however, when this does not occur, serious legal and ethical consequences may result. This study aims to describe how informed consent to medical procedure is being perceived by patients and doctors in PNG. Culture plays a key role in the understanding of how consent should be given. The restrictions given by religion, family, spouses, and custom are some of those aspects that both doctors and patients perceive to hinder medical treatment.

Another factor is the lack of knowledge of patients in terms of their rights when seeking treatment. They feel inferior when seeking medical treatment from the doctor. Both in the survey and focus group discussion, patients have perceived consent forms are for doctor's rights only and that they do not see the consent forms as important.

At the doctors' end, they seemed to lack knowledge on the legalities of informed consent. They do not know if there is (are) any law(s) directly concerning how informed consent should be asked for or what general guidelines they can use or employ in their practice. They admitted that they felt lack of connection with the patients. It seems they feel that it is just a habit of diagnosis, giving medicines, procedures and then leave.

Results found in PNG are no different to India and 


\section{Consent to Medical Procedures in Papua New Guinea}

Japan. Lack of information on the rights among patients may pose them danger. This is also true for doctors, they should be aware on how to handle patients of differing cultures and beliefs. But most of all, there is a need for a policy on how informed consent should be asked for and given. This will guide patients and doctors in their functions to help each other build their communication, and advance the interests of both patient and the doctor.

\section{References}

[1] Berg, J. W., Applebaum, P. S., and Lidz, C. W. 2001. Informed Consent: Legal Theory and Clinical Practice. Oxford University Press, 1-340.

[2] Faden, R. R., and Beauchamp, T. L. 1986. A History and Theory of Informed Consent. New York: Oxford University Press, 1-392.

[3] Belmont Report. 1979. The Belmont Report: Ethical Principles and Guidelines for the Protection of Human Subjects of Research. Retrieved from: http://www.hhs. gov/ohrp/humansubjects/guidance/belmont.html.
[4] SCR No. 2 of 1984: Re Medical Privilege (1985) PNGLR 247.

[5] SCR No. 3 of 1992 (1992) PNGLR 114.

[6] PNG's Constitution, Section 49 "Right to Privacy".

[7] Chima, S. C. 2013. "Evaluating the Quality of Informed Consent and Contemporary Clinical Practices by Medical Doctors in South Africa: An Empirical Study.” Presented at the 3rd Ethics, Human Rights and Medical Law Conference, Africa Health Congress 2013, Johannesburg, South Africa, 7-9 May 2013.

[8] Ashraf, B., Tasnim, N., Saaiq, M., and Zaman, K. U. 2014. "An Audit of the Knowledge and Attitudes of Doctors towards Surgical Informed Consent (SIC).” Int. J. Health Policy Manag. 3: 315-21.

[9] Rajesh, D. R., Singh, A., Chopra, M., Singh, G., Venkteshan, M., Bhardwaj, A., Kaur, B., and Aggarwal, O. P. 2013. "Patients Awareness, Attitude, Understanding and Perceptions towards Legal Nature of Informed Consent.” J Indian Acad Forensic Med. 35 (1): 0971-3.

[10] Parmar, P., Rathod, B., Rathod, S., and Parikh, A. 2016. "Consent in Medical Practice-Perceptions of Patients towards Legal Aspects of Informed Consent.” IAIM 3 (4): 105-10. 